\title{
Characteristics of adults and children diagnosed with tuberculosis in Lilongwe, Malawi: findings from an integrated HIV/TB clinic
}

\author{
C. Feldacker ${ }^{1,2}$, H. Tweya ${ }^{1,3}$, O. Keiser ${ }^{4}$, R. Weigel ${ }^{5}$, M. Kalulu ${ }^{1}$, L. Fenner ${ }^{4}$, M. Egger $^{4}$, E. \\ Manda $^{1}$, J. B. Mwafilaso ${ }^{1}$, C. Kamba ${ }^{6}$, and S. Phiri ${ }^{1}$ \\ ${ }^{1}$ Lighthouse Trust, Lilongwe, Malawi ${ }^{2}$ International Training and Education Center for Health, \\ University of Washington, Seattle, USA ${ }^{3}$ The International Union Against Tuberculosis and Lung \\ Disease, Paris, France ${ }^{4}$ Institute of Social and Preventive Medicine, University of Bern, \\ Switzerland ${ }^{5}$ Liverpool School of Tropical Medicine, UK ${ }^{6}$ Lilongwe District Health Office, Malawi
}

\begin{abstract}
Objectives-To describe initial registration characteristics of adult and pediatric TB patients at a large, public, integrated TB and HIV clinic in Lilongwe, Malawi, between January 2008 December 2010.

Methods-Routine data on TB patient category and TB type, stratified by HIV and ART status, were used to explore differences in proportions among TB-only, TB/HIV co-infected patients not on ART, and TB/HIV co-infected patients on ART using Chi-square tests.. Trends over time illustrate strengths and weaknesses of integrated service provision.

Results-Among 10,143 adults, HIV ascertainment and ART uptake were high and increased over time. The proportion of relapse was highest among those on ART (5\%). The proportion of smear-positive pulmonary TB (PTB) was highest among HIV-negative TB patients (34.9\%); extra-pulmonary TB (EPTB) was lowest among TB-only (16.2\%). Among 338 children $<15$ years, EPTB and smear-positive PTB were more common among TB-only patients. Time trends showed significant increases in the proportion of adults with smear-positive PTB and the proportion of adults already on ART before starting TB treatment. However, some co-infected patients still delay ART initiation.
\end{abstract}

Conclusions-HIV ascertainment and ART uptake among co-infected patients is successful and improving over time. However, delays in ART initiation indicate some weakness linking TB/HIV patients into ART during TB follow-up care. Improved TB diagnostics and screening efforts, especially for pediatric patients, may help improve quality care for co-infected patients. These results may aid efforts to prioritize TB and HIV prevention, education, and treatment campaigns for specific populations.

\section{Keywords}

TB/ART service integration; Malawi; TB; ART; HIV; smear-positive PTB 


\section{Introduction}

Malawi, similar to its sub-Saharan neighbors, faces the burden of fighting simultaneous HIV and TB epidemics. In 2009, Malawi reported 37,000 TB cases to the WHO with a case detection rate of $49 \%$ of smear-positive infections (WHO 2011), below the STOP TB target of 70\% smear-positive case detection (WHO 2008). HIV prevalence in Malawi, is $12 \%$ : 323,638 people were alive on ART ( $62 \%$ female) as of December, $30^{\text {th }}, 2011$, representing approximately 65\% ART coverage nationwide (Government of Malawi Ministry of Health 2011). Among TB patients, approximately $67 \%$ are HIV co-infected (Government of Malawi Ministry of Health 2011); 43\% of newly diagnosed TB cases are HIV-infected and already on ART (Ministry of Health and Population Malawi 2011).

Previous studies from the region describe TB prevention, screening or treatment among HIV-infected patients (Abdool Karim et al. 2010; Harries et al. 2010; Khan et al. 2010; Lawn et al. 2006; Lawn \& Wood 2011) and highlight efforts at TB and HIV integration (Harries et al. 2006; Harries et al. 2002a; Harries et al. 2004b; Uyei et al. 2011), including within Malawi (Chimzizi \& Harries 2007; Harries et al. 2004a, 2009a; Libamba et al. 2005, 2006; Zachariah et al. 2005). However, fewer studies describe patient demographic characteristics, including HIV testing and ART uptake, from the perspective of TB care settings (Chimzizi et al. 2004; Lawn et al. 2011a,b) that may serve as an entry point of care for co-infected patients. To the authors' knowledge, no recent study describes TB patient characteristics at TB treatment initiation based on both HIV and ART status, including those who are not HIV co-infected.

Therefore, we aim to fill a gap in the literature and complement previous research by describing baseline characteristics, HIV ascertainment, and ART status of adult and pediatric patients at TB treatment registration within an integrated HIV/TB care setting. Lighthouse Trust's Martin Preuss Centre (MPC) is the largest integrated public TB/HIV clinic in Malawi, providing an opportunity to describe patient characteristics that are expected to be different at an integrated site, including more complete HIV status ascertainment and ART information at TB registration. We use routine, programmatic, TB case data from MPC between January 2008 - December 2010 to explore differences in TB patient category as defined by the National TB Control Program (NTP) (new, relapse, failure, retreatment after default, other) and TB type (extrapulmonary (EPTB) and pulmonary (PTB)) among three patient categories: 1) non-HIV-infected TB patients (TBonly); 2) HIV-infected TB patients not on ART at TB treatment initiation (TB/HIV); and 3) HIV-infected TB patients already on ART prior to TB treatment initiation (TB/ART). Trends over the 3-year time period were examined to help provide critical information for program and policy planning. The results from this descriptive study may aid understanding about the current TB epidemic, inform future TB/HIV integration efforts, and lead to improvements in TB control programs and policies.

\section{Methods}

Setting

Lighthouse Trust works in close coordination with the Ministry of Health $(\mathrm{MoH})$ and District Health Office (DHO) to operate two large integrated HIV testing, treatment and care clinics in Lilongwe, Malawi: Lighthouse Clinic (LH) on the campus of Kamuzu Central Hospital and the Martin Preuss Center (MPC) located near the central bus station on the campus of Bwaila Maternity Hospital. Combined, Lighthouse is the largest provider of ART in Malawi's central region with over 16,000 patients alive on ART: approximately 7,500 at LH and 8,500 at MPC. LH and MPC screen all patients for TB at each clinic visit and 
provide TB treatment; neither clinic conducts intensive TB case finding activities. LH cares only for HIV-infected patients.

MPC opened in late 2006 as a purposeful TB/HIV integrated care clinic. In addition to its HIV-related services, MPC currently houses the largest TB registration centre in Malawi with approximately 3,500 TB patients and 3900 TB suspects, annually. Nationwide, there are 70 TB registration sites including 20 in Central Region and 5 in Lilongwe District (Government of Malawi Ministry of Health 2011). Patients must register and begin treatment at one of these sites but may elect to continue TB treatment at the registration site or another peripheral health facility. MPC's integrated TB/HIV model was described in detail previously (Phiri et al. 2011).

At MPC's TB treatment registration, more than 95\% of TB patients have known HIV status at TB treatment initiation in large part due to its opt-out HIV testing policy for TB suspects and patients. All HIV-positive TB patients are eligible to start ART according to national guidelines, and co-infected patients receive both TB and HIV clinical care at MPC. Between 50-60\% of HIV-positive TB patients initiate ART and complete their TB treatment at MPC. Other patients initiate TB treatment at MPC but complete TB treatment at one of 18 peripheral sites in Lilongwe. The ART status of patients who do not initiate ART at MPC is unknown.

At MPC, as with other public TB clinics in Malawi, diagnosis of TB for adults and children is based most commonly on clinical examination, sputum smear microscopy, and chest radiography. Extrapulmonary (EPTB) may also be diagnosed bacteriogically or histopathologically. The Malawi NTP control program classifies patients into 5 patient categories (Malawi Ministry Of Health 2007): 1) new: patients with no previous TB treatment; 2) relapse: cured or completed treatment prior to becoming smear-positive; 3 ) failure: remained sputum smear-positive at five months or more during first-line treatment; 4) re-treatment after default (RAD): interrupted treatment for more than two months and returned with smear-positive TB; and 5) other, which may include chronic re-treatment TB patients and patients with recurrent smear-negative pulmonary TB (Tweya et al. 2011). Once diagnosed with TB, patient details are recorded in the national TB registers by the district TB officer. New and re-treatment TB cases are treated in accordance with standard WHO regimens (WHO 2003) consisting of rifampicin (R), isoniazid (H), pyrazinamide (Z) and ethambutol (E).

\section{Study design and population}

This retrospective cohort study of all TB patients used routine program data from both TB registers and patient treatment cards collected from January 2008 to December 2010 at MPC. All adults and children at MPC diagnosed with active TB according to national guidelines were eligible.

\section{Data collection}

All patient demographic and ART-related information was extracted from MPC's real-time electronic data system (EDS). TB data from paper registers, including TB registration number; registration date; TB type (EPTB or PTB); patient category; initial sputum microscopy results; HIV status (positive, negative, unknown); ART status (ART before TB treatment; ART at or after TB treatment initiation; not on ART) and treatment regimen, was back entered into an offline Access database and matched with demographic and ART information using patient identification codes. Missing or incomplete TB data in the register were filled or verified using individual patient mastercards. CD4 test results were included

Trop Med Int Health. Author manuscript; available in PMC 2013 September 10. 
from the EDS when available using the closest recorded CD4 test within 90 days of TB registration.

\section{Data Analysis}

Data analysis, stratified by age (children $<15$ years and adults $\geq 15$ years) was conducted using Access 2003 and STATA 9.2 (StataCorp 2005). Frequency tables and univariate analysis detected problems in the dataset, issues of linearity, or unusual outliers; data clerks corrected inconsistencies. Chi square tests of proportions were used to determine differences over time and between the three groups across variables of interest: 1 ) non-HIV-infected TB patients (TB-only); 2) HIV-infected TB patients not on ART at TB treatment initiation (TB/ HIV); 3) HIV-infected TB patients already on ART prior to TB treatment initiation (TB/ ART). Statistical significance was defined as $p \leq 0.05$.

\section{Ethics approval}

The Malawi National Health Science Research Committee provided general blanket approval for the collection and use of routine programmatic data for monitoring and evaluation at Lighthouse Clinics for 2011, including this study.

\section{Results}

\section{Adults $\geq 15$ years}

Of the 10,595 adult TB patients aged 15 or older, $5985(59 \%)$ were male. The largest proportion of patients was between the ages of 25-34 (38.4\%). Most patients (89.5\%) were new TB cases. Of the 10,143 patients (96\%) with recorded HIV information, $60.2 \%$ were tested before TB treatment. The majority $(66.5 \%)$ of adult TB patients were HIV coinfected: $64 \%$ of men and 70\% of women. Among the 6747 HIV+ patients, $3071(44.7 \%)$ had a recorded ART start date: $39.5 \%$ started before TB treatment, $55.6 \%$ started during TB treatment, and $4.9 \%$ started after TB treatment. Among HIV+ patients, only $792(11.7 \%)$ had a recorded CD4 count within 90 days of TB registration. Of all adults, $48.6 \%$ chose to start and complete TB treatment at MPC. Among TB/ART patients, 2228 (72.5\%) were managed at MPC. The number of TB patients managed at MPC decreased over time: 4271, 3263 , and 3061 adult patients registered in 2008, 2009, and 2010, respectively.

Patient demographics differed between the TB-only, TB/HIV, and TB/ART groups (Table 1). Men comprised $63 \%$ of the TB-only group; $58.4 \%$ of TB/HIV; and $50.2 \%$ of TB/ART patients $(\mathrm{p}<0.001)$. Age varied significantly across groups $(\mathrm{p}<0.001)$. TB-only patients were more likely to be under $25(20.9 \%)$ while a higher proportion of TB/HIV (73.2\%) and TB/ART (73.4\%) patients were concentrated in the middle age groups, ages 25-45, as compared to TB-only (47\%). TB-only patients were also more likely to be 45 years or older (32.2\%) as compared to TB/HIV (16.2\%) and TB/ART (17.6\%) patients.

Relapse was higher among TB/ART (5.0\%) than TB-only patients (3.2\%) and TB/HIV patients $(2.6 \%)(\mathrm{p}<0.001)$. TB/ART patients had the highest proportion of "other" patients $(13 \%)(\mathrm{p}<0.001)$, patients who are more likely to be mis-categorized re-treatment cases and HIV co-infected (Tweya et al. 2011). EPTB was most common among TB/ART (26.9\%) and less common among TB/HIV (20.7\%) and TB-only (16.2\%) (p < 0.001). Smear-positive PTB was significantly higher among TB-only patients (34.9\%) than those co-infected (p $<0.001$ ). Across all 3 groups, approximately $50 \%$ were smear-negative PTB. Among those with CD4, there was no significant relationship between TB classification and CD4 count $(\mathrm{p}=0.532)($ data not shown $)$. 
Considering trends over time (Table 3$)$, TB classification varied over time $(\mathrm{p}<0.001)$ : the proportion of smear-positive PTB increased from $24.6 \%$ to $37.0 \%$ of all patients over time. EPTB cases also rose slightly from $18.8 \%$ to $22.5 \%$ over the same 3 -year period. According to the standard ARV status categories at TB registration, the proportion of TB/ART patients increased significantly ( $\mathrm{p}<0.001)$ between $2008(19.3 \%)$ and $2010(48.6 \%)$. Among the $74 \%$ of patients with a specific ART start date $(\mathrm{N}=2,229)$, there were significant changes in the timing of ART initiation. Between 2008 and 2010, those started on ART within 30 days of TB treatment increased from $1.4 \%$ to $57.0 \%$ while the proportion that started ART 60 or more days after TB treatment initiation dropped dramatically from $67.7 \%$ to $10.3 \%$ $(\mathrm{p}<0.001)$. HIV co-infection $(\mathrm{p}=0.592)$ and gender $(\mathrm{p}=0.789)$ did not vary significantly over time.

\section{Children $<15$ years}

Of the 364 children, 338 children had HIV status information and were included in this analysis (Table 2). Of 338, 49.7\% were tested before seeking TB treatment and $43.4 \%$ of all tested were HIV-positive. About half (49.7\%) were male and 29.3\% were under age 5. Most children (94.4\%) were new TB patients; $4.8 \%$ were classified as "other". Among all 299 with known TB classification, $18.1 \%$ were diagnosed with EPTB, $69.9 \%$ with smearnegative PTB, and 12\% had smear-positive PTB. Among the 148 HIV+ patients, 66 (44.6\%) had a recorded ART start date: 15 children started ART before TB treatment, 50 children started ART during TB treatment, and one child started ART after TB treatment. Among $\mathrm{HIV}+$ patients, 25 had a recorded CD4 count within 90 days of TB registration. Of all pediatric patients, 58.9\% started and completed TB treatment at MPC; among TB/ART patients, $55(83.3 \%)$ were managed at MPC. The number of pediatric TB patients varied over time: 151,72, and 115 patients registered in 2008, 2009, and 2010, respectively.

Among children, TB baseline characteristics differed among TB-only, TB/HIV, and TB/ ART groups. EPTB was highest among children with TB-only (20.7\%), as compared to $14.7 \%$ of TB/HIV and $14.3 \%$ of TB/ART patients. Smear-negative PTB was the most common type of TB across all groups: $62 \%$ of TB-only; $81 \%$ of TB/HIV and $79 \%$ of TB/ ART children. Smear positive TB was less frequent with $17.8 \%$ of TB-only as compared to $4.3 \%$ of TB/HIV and $7.1 \%$ of TB/ART children. There were no other significant differences among children based on their TB, HIV, and ART status nor significant trends over time (Table 3).

\section{Discussion}

To our knowledge, this is one of the first papers to describe the treatment initiation characteristics of both adult and pediatric TB patients at a large, public, integrated TB/HIV management setting, including exploration of trends over time. We note several differences between TB patient groups based on TB type and patient category as well as by HIV and ART status.

First, this study shows both strengths and weaknesses in TB/HIV integration at MPC over time. HIV ascertainment was highly successful: during this 3-year period, the proportion with known HIV status remained between 95-97\%. In comparison, in 2011 the national HIV ascertainment rate among TB patients was ranged from 83-90\% (Government of Malawi Ministry of Health 2011). The consistency of HIV ascertainment demonstrates MPC's successful integration of HIV testing and lends further support for a provider-initiated, optout HIV testing approach within TB care settings (Harries et al. 2009b). Time trends among adults also reveal significant increases in the proportion of patients already on ART, a positive finding that likely reflects both MPC's emphasis on TB screening among ART patients as well as the nation-wide scale up of ART (Ministry of Health and Population 
Malawi 2011). There was also a significant and positive increase in earlier initiation of coinfected patients onto ART: between 2008 and 2010, the proportion of co-infected adults at MPC who started ART within 30 days of TB treatment initiation increased dramatically from $1.4 \%$ to $57 \%$. This shift in treatment timing was likely influenced by results from research on early mortality among TB/HIV patients in Malawi (Zachariah et al. 2007) and subsequent guideline changes that recommended earlier ART initiation among those coinfected (Ministry of Health Malawi 2008). Despite successes, some TB patients still delay ART initiation. In 2008, more than $67 \%$ of co-infected patients delayed ART until after the intensive phase, 60 days, after TB treatment; by 2010, only $10.3 \%$ of co-infected patients started as late. Follow-up exploration (not shown) found that the 78 patients, or $10 \%$, who did not start ART within 60 days were more likely to be male $(72 \%)(\mathrm{p}<0.05)$ and within the age group 25-34 years $(47 \%)(\mathrm{p}<0.05)$. Although significant progress is clear, continued delayed uptake may indicate persistent weaknesses in linking TB/HIV patients into ART during TB follow-up care as well as continued patient hesitancy or fears about concurrent treatment (Phiri et al. 2011) which merit attention. Lastly, the overall proportion of TB patients with smear-positive TB increased over time. No new laboratory technology or other systematic changes were implemented at MPC during this period, and it is unknown whether case detection improved or whether there was an actual increase in the proportion of smearpositive patients.

Second, MPC's integrated service model also allows for identification of differences in TB patient category that exist based on HIV and ART status. First, as expected, the proportion of new TB patients, as opposed to all other categories, was highest among TB-only patients. Second, and more interestingly, the proportion of relapse among MPC patients was highest among those on ART: $3.2 \%$ of TB-only, $2.6 \%$ of TB/HIV, and 5\% of TB/ART patients were categorized as relapse. Although distinguishing between relapse and re-infection would require molecular diagnostics beyond the capability of MPC, this finding is interesting in comparison to a study on recurrent TB in Karonga, Malawi. Crampin et al. (2010) found no significant difference in proportions of relapse among patients based on HIV status, but found re-infection highest among those TB/HIV co-infected. The role of ART was not considered in the Karonga study, but it may have played a role among MPC patients. Among MPC patients, immune reconstitution inflammatory syndrome (IRIS) or ARTassociated TB may have played a role in development or redevelopment of active TB among HIV-infected patients (Lawn et al. 2008); 94 MPC ART patients developed active TB within 6 weeks of starting ART. Moreover, lack of adherence to either TB or ART regimens, due to increased pill burdens, side effects, or perceptions of regimen complexity on the part of patients or clinicians, may increase the likelihood of TB recurrence among co-infected patients (Panjabi et al. 2007). No additional information on treatment adherence or TB recurrence was available at the time of the study.

Third, TB patient baseline characteristics differ based on HIV and ART status. First, the proportion of smear-positive PTB was highest among TB-only patients (34.9\%) as HIV coinfected patients are more likely to have smear-negative PTB or EPTB (Harries 1990; Shafer et al. 1991) while smear-negative PTB was similar across all patient groups. Also the proportion of EPTB was higher among TB/HIV (20.7\%) and TB/ART (26.9\%) patients than among TB-only adults (16.2\%), confirming previous research (Corbett et al. 2006). In post hoc analysis considering whether time on ART was a factor in predicting EPTB vs. PTB classification (data not shown), no significant association between time on ART and TB classification was found. Lastly, although gender did not vary significantly between groups nor show changes over time, women are the minority of TB-only (37\%); TB/HIV (41.6\%) and TB/ART (49.8\%) patients at MPC although $62 \%$ of nationwide ART patients are female (Government of Malawi Ministry of Health 2011) confirming previous studies on the disproportionate number of men infected with TB in Malawi (Boeree et al. 2000). 
Fourth, our study contributes information on TB characteristics among children, complementing and updating previous research in Malawi (Harries et al. 2002b,1997; Kiwanuka et al. 2001; Poerksen et al. 2008). Among MPC children, boys and girls are infected in equal proportions suggesting household infection in contrast to adult men who may face external occupational risk areas outside of the home. Also, the vast majority of children in all categories were smear-negative PTB: $61.5 \%$ of TB-only; $81.0 \%$ of TB/HIV, and $78.6 \%$ of TB/ART children. The proportion of EPTB also differed between groups: $20.7 \%$ of TB-only pediatric patients as compared to about $14 \%$ of co-infected children regardless of ART status. These findings may be muddled by the inherent complications of collecting sputum from young children (Zar et al. 2005) and may indicate the need for improved diagnostic tools such as GenXpert that are currently unavailable at MPC.

Screening of children could also improve if smear-positive, adult TB patients brought in household contacts, including children, for testing and preventative treatment as recommended by the NTP. However, funding constraints prevent or delay active follow-up of smear-positive patients, limiting the effectiveness of efforts to combat household exposure. Lastly, time trends of increased proportions of patients on ART before TB treatment apparent among adults is not replicated among children, possibly suggesting that there are weaknesses in TB screening among children on ART or that TB-IRIS may be less common.

\section{Limitations}

This descriptive study should be considered with the following limitations. Among all patients, 478 (4.4\%), 26 children and 451 adults, did not have HIV status information and were excluded from the baseline characteristics analysis but included in the trends over time. Also, some discrepancies in the paper-based records could not be reconciled, excluding 73 patients. There were few patients with CD4 results and ART adherence data, preventing consideration of these factors. Also, many patients who started ART at a clinic other than MPC did not provide an exact ART start date, potentially biasing the time trends results of ART initiation timing. Furthermore, we grouped patients who were on ART for any length of time before TB treatment, reducing the ability to determine if time on ART could have played a role in TB characteristics at TB treatment registration. Lastly, although MPC is Malawi's largest TB registration site, the findings may be most useful for other large burden, integrated TB/ART clinics in urban areas.

\section{Conclusions}

We described patient characteristics at TB registration at MPC, the largest integrated public TB/HIV clinic in Malawi, among three groups: TB-only; HIV-infected TB patients not on ART (TB/HIV); and HIV-infected TB patients already on ART prior (TB/ART). We found that new TB patients were more likely to be TB-only patients while the proportion of relapse was highest among TB/ART patients. Smear-positive TB was highest among TB-only while the proportion of EPTB was higher among both co-infected groups. Most children were smear-negative PTB with higher proportion of TB-only children diagnosed with EPTB. Time trends showed consistently high ascertainment of HIV status among TB registrants and significant improvements in early ART initiation among those co-infected.

In response to the study findings and to strengthen future research, MPC is undertaking several steps to improve quality care. In early 2012, the established electronic data system (EDS) for ART will be expanded to include critical information for management of patients infected with TB, including HIV/TB co-infected patients. Not only will the EDS component reduce the occurrence of missing data, but the system will include reminders for providers to initiate co-infected patients not yet on ART and include TB patient contact information for follow-up testing and treatment for household members, especially children. MPC is also 
conducting additional refresher training with professional staff and increasing both monitoring and supervision efforts to continue improving the integrated care model.

These findings also suggest several areas for additional operations research at MPC or other low-resource, integrated care settings. First, delays in ART initiation among some patients are a cause for concern and weaknesses in the linkages between TB and ART treatment initiation should be examined. Second, the increase in the proportion of patients with smearpositive TB suggests that increased efforts at infection control may be necessary to reduce the spread of TB within the integrated clinic setting. Third, future quantitative studies should look at the associations between TB and ART treatment adherence in the development and diagnosis of TB as well as examine factors that influence ART initiation and uptake among co-infected patients. Lastly, exploration of the factors that influence ART uptake and timing among co-infected patients would add considerably to understanding of the drivers of and deterrents to quality, integrated care.

\section{References}

Abdool Karim SS, Naidoo K, Grobler A, et al. Timing of initiation of antiretroviral drugs during tuberculosis therapy. New England Journal of Medicine. 2010; vol. 362(no. 8):697-706. [PubMed: 20181971]

Boeree MJ, Harries AD, Godschalk P, et al. Gender differences in relation to sputum submission and smear-positive pulmonary tuberculosis in Malawi Notes from the Field. The International Journal of Tuberculosis and Lung Disease. 2000; vol. 4(no. 9):882-884. [PubMed: 10985659]

Chimzizi R, Gausi F, Bwanali A, et al. Voluntary counselling, HIV testing and adjunctive cotrimoxazole are associated with improved TB treatment outcomes under routine conditions in Thyolo District, Malawi. The International Journal of Tuberculosis and Lung Disease. 2004; vol. 8(no. 5):579-585. [PubMed: 15137534]

Chimzizi R, Harries A. Joint tuberculosis/HIV services in Malawi: progress, challenges and the way forward. Bulletin of the WHO. 2007; vol. 85(no. 5):385-386.

Corbett EL, Marston B, Churchyard GJ, De Cock KM. Tuberculosis in sub-Saharan Africa: opportunities, challenges, and change in the era of antiretroviral treatment. Lancet. 2006; vol. 367(no. 9514):926-937. [PubMed: 16546541]

Crampin AC, Mwaungulu JN, Mwaungulu FD, et al. Recurrent TB: relapse or reinfection? The effect of HIV in a general population cohort in Malawi. AIDS (London, England). 2010; vol. 24(no. 3): 417.

Government of Malawi Ministry of Health. Integrated HIV Program Report October - December 2011. HIV / AIDS Unit, Department of Clinical Services; Lilongwe, Malawi: 2011.

Harries AD. Tuberculosis and human immunodeficiency virus infection in developing countries. Lancet. 1990; vol. 335(no. 8686):387-390. [PubMed: 1968123]

Harries AD, Boxshall M, Phiri S, Kwanjana J. Managing HIV and tuberculosis in sub-Saharan Africa. Lancet. 2006; vol. 367(no. 9525):1817-1818. [PubMed: 16753481]

Harries AD, Hargreaves NJ, Chimzizi R, Salaniponi FM. Highly active antiretroviral therapy and tuberculosis control in Africa: synergies and potential. Bulletin of the WHO. 2002a; vol. 80(no. 6): 464-469.

Harries AD, Hargreaves NJ, Graham SM, et al. Childhood tuberculosis in Malawi: nationwide casefinding and treatment outcomes. The International Journal of Tuberculosis and Lung Disease. 2002b; vol. 6(no. 5):424-431. [PubMed: 12019918]

Harries AD, Libamba E, Schouten EJ, et al. Expanding antiretroviral therapy in Malawi: drawing on the country's experience with tuberculosis. British Medical Journal. 2004a; vol. 329(no. 7475): 1163-1166. [PubMed: 15539674]

Harries AD, Libamba E, Schouten EJ, et al. Expanding antiretroviral therapy in Malawi: drawing on the country's experience with tuberculosis. BMJ. 2004b; (no. 329):1163-1166. [PubMed: 15539674] 
Harries AD, Parry C, Mbewe LN, et al. The pattern of tuberculosis in Queen Elizabeth Central Hospital, Blantyre, Malawi: 1986-1995. The International Journal of Tuberculosis and Lung Disease. 1997; vol. 1(no. 4):346-351. [PubMed: 9432391]

Harries AD, Zachariah R, Corbett EL, et al. The HIV-associated tuberculosis epidemic: when will we act? The Lancet. 2010; vol. 375(no. 9729):1906-1919.

Harries AD, Zachariah R, Jahn A, Schouten EJ, Kamoto K. Scaling Up Antiretroviral Therapy in Malawi-Implications for Managing Other Chronic Diseases in Resource-Limited Countries. JAIDS Journal of Acquired Immune Deficiency Syndromes. 2009a; vol. 52:S14-S16. 10.1097/ QAI.1090b1013e3181bbc1099e.

Harries AD, Zachariah R, Lawn SD. Providing HIV care for co-infected tuberculosis patients: a perspective from sub-Saharan Africa [State of the art series. Tuberculosis. Edited by ID Rusen. Number 3 in the series]. The International Journal of Tuberculosis and Lung Disease. 2009b; vol. 13(no. 1):6-16. [PubMed: 19105873]

Khan FA, Minion J, Pai M, et al. Treatment of active tuberculosis in HIV-coinfected patients: a systematic review and meta-analysis. Clinical Infectious Diseases. 2010; vol. 50(no. 9):12881299. [PubMed: 20353364]

Kiwanuka J, Graham SM, Coulter JBS, et al. Diagnosis of pulmonary tuberculosis in children in an HIV-endemic area, Malawi. Annals of Tropical Paediatrics: International Child Health. 2001; vol. 21(no. 1):5-14.

Lawn SD, Campbell L, Kaplan R, et al. Delays in starting antiretroviral therapy in patients with HIVassociated tuberculosis accessing non-integrated clinical services in a South African township. BMC infectious diseases. 2011a; vol. 11(no. 1):258. [PubMed: 21957868]

Lawn SD, Fraenzel A, Kranzer K, et al. Provider-initiated HIV testing increases access of patients with HIV-associated tuberculosis to antiretroviral treatment. SAMJ: South African Medical Journal. 2011b; vol. 101(no. 4):258-262.

Lawn SD, Myer L, Bekker LG, Wood R. Burden of tuberculosis in an antiretroviral treatment programme in sub-Saharan Africa: impact on treatment outcomes and implications for tuberculosis control. Aids. 2006; vol. 20(no. 12):1605. [PubMed: 16868441]

Lawn SD, Wilkinson RJ, Lipman MCI, Wood R. Immune reconstitution and" unmasking" of tuberculosis during antiretroviral therapy. American Journal of Respiratory and Critical Care Medicine. 2008; vol. 177(no. 7):680. [PubMed: 18202347]

Lawn SD, Wood R. Tuberculosis in antiretroviral treatment services in resource-limited settings: addressing the challenges of screening and diagnosis. Journal of Infectious Diseases. 2011; vol. 204(no. suppl 4):S1159-S1167. [PubMed: 21996698]

Libamba E, Makombe S, Harries AD, et al. Scaling up antiretroviral therapy in Africa: learning from tuberculosis control programmes - the case of Malawi. International Journal of Tuberculosis and Lung Disease. 2005; vol. 9(no. 10):1062-1071. [PubMed: 16229216]

Libamba E, Makombe S, Mhango E, et al. Supervision, monitoring and evaluation of nationwide scale-up of antiretroviral therapy in Malawi. Bulletin of the WHO. 2006; vol. 84(no. 4):320-326.

Malawi Ministry Of Health. Malawi National Tuberculosis Control Programme Manual. MoH; Lilongwe: 2007.

Ministry of Health and Population Malawi. ART in the public sector in Malawi, results up to 1st June 2011. 2011.

Ministry of Health Malawi. [viewed April 16, 2012] Treatment of AIDS: Guidelines for the use of antiretroviral therapy in Malawi. Third Edition2008. <http://www .hivunitmohmw.org/Main/ AntiretroviralTherapy>

Panjabi R, Comstock GW, Golub JE. Recurrent tuberculosis and its risk factors: adequately treated patients are still at high risk [Review Article]. The International Journal of Tuberculosis and Lung Disease. 2007; vol. 11(no. 8):828-837. [PubMed: 17705947]

Phiri S, Khan PY, Grant AD, et al. Integrated tuberculosis and HIV care in a resource limited setting: experience from the Martin Preuss centre, Malawi. Tropical Medicine \& International Health. 2011; vol. 16(no. 11):1397-1403. [PubMed: 21806742]

Poerksen G, Kazembe PN, Graham SM. Challenges of Childhood TB/HIV Management in Malawi. Malawi Medical Journal. 2008; vol. 19(no. 4):142-148. 
Shafer RW, Kim DS, Weiss JP, Quale JM. Extrapulmonary tuberculosis in patients with human immunodeficiency virus infection. Medicine. 1991; vol. 70(no. 6):384. [PubMed: 1956280]

Tweya H, Kanyerere H, Ben-Smith A, et al. Re-Treatment Tuberculosis Cases Categorised as "Other": Are They Properly Managed? PLoS One. 2011; vol. 6(no. 12):e28034. [PubMed: 22194804]

Uyei J, Coetzee D, Macinko J, Guttmacher S. Integrated delivery of HIV and tuberculosis services in sub-Saharan Africa: a systematic review. The Lancet Infectious Diseases. 2011; vol. 11(no. 11): 855-867. [PubMed: 22035614]

World Health Organisation. Treatment of Tuberculosis: guidelines for national programmes. Geneva: 2003.

WHO. Tuberculosis detection rate under DOTS (percentage) 2011.2008. <http://www.who.int/ whosis/indicators/compendium/2008/4tdr/en/index.html>

WHO. TB Country Profile; Malawi: 2011.2011<https://extranet.who.int/sree/Reports? op=Replet\&name=\%2FWHO_HQ_Reports $\% 2$ FG2\%2FPROD \%2FEXT $\%$ 2FTBCountryProfile \&ISO2=MW\&outtype $=$ html>

Zachariah R, Fitzgerald M, Massaquoi M, et al. Does antiretroviral treatment reduce case fatality among HIV-positive patients with tuberculosis in Malawi? The International Journal of Tuberculosis and Lung Disease. 2007; vol. 11(no. 8):848-853. [PubMed: 17705949]

Zachariah R, Teck R, Ascurra O, et al. Can we get more HIV-positive tuberculosis patients on antiretroviral treatment in a rural district of Malawi? International Journal of Tuberculosis and Lung Disease. 2005; vol. 9(no. 3):238-247. [PubMed: 15786885]

Zar HJ, Hanslo D, Apolles P, Swingler G, Hussey G. Induced sputum versus gastric lavage for microbiological confirmation of pulmonary tuberculosis in infants and young children: a prospective study. The Lancet. 2005; vol. 365(no. 9454):130-134. 
Table 1

Differences in proportions between adult TB-only, TB/HIV, and TB/ART patients

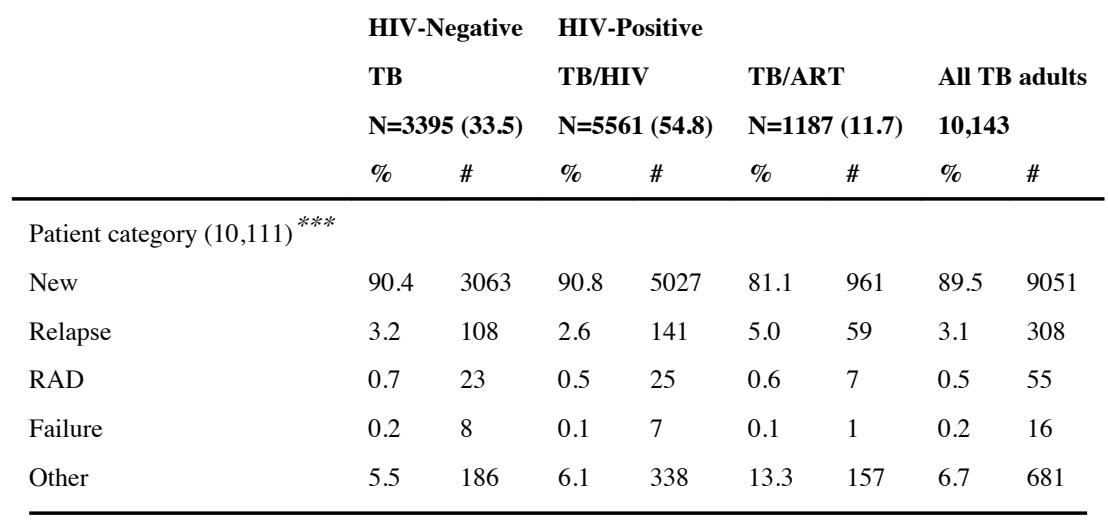

TB Classification $(9,889) * * *$

\begin{tabular}{lllllllll} 
EPTB & 16.2 & 541 & 20.7 & 1117 & 26.9 & 312 & 19.9 & 1970 \\
Smear-negative PTB & 48.9 & 1628 & 55.7 & 3005 & 50.0 & 582 & 52.7 & 5215 \\
Smear-positive PTB & 34.9 & 1163 & 23.1 & 1272 & 27.3 & 269 & 27.3 & 2704 \\
\hline
\end{tabular}

TB treatment $(4,546) * * *$

\begin{tabular}{|c|c|c|c|c|c|c|c|c|}
\hline RHZE & 94.2 & 1326 & 93.8 & 2204 & 88.1 & 685 & 92.9 & 4225 \\
\hline Isoniazid & 0.3 & 4 & 0.3 & 6 & 0.1 & 1 & 0.2 & 11 \\
\hline ZHRS & 0.1 & 1 & 0.1 & 2 & 0.1 & 1 & 0.1 & 4 \\
\hline RHZE+S & 5.4 & 76 & 5.2 & 122 & 10.5 & 83 & 6.2 & 281 \\
\hline $\mathrm{RHZ}+\mathrm{s}$ & 0 & 0 & 0.7 & 16 & 1.1 & 9 & 0.6 & 25 \\
\hline \multicolumn{9}{|c|}{ Gender ${ }^{* * *}$} \\
\hline Male & 63.0 & 2139 & 58.4 & 3250 & 50.2 & 596 & 59.0 & 5985 \\
\hline Female & 37.0 & 1256 & 41.6 & 2311 & 49.8 & 591 & 41.0 & 4158 \\
\hline \multicolumn{9}{|l|}{$\mathrm{Age}^{* * *}$} \\
\hline $15-24$ & 20.9 & 709 & 10.6 & 589 & 9.0 & 107 & 13.9 & 1405 \\
\hline $25-34$ & 30.1 & 1021 & 43.2 & 2401 & 40.2 & 477 & 38.4 & 3899 \\
\hline $35-44$ & 16.9 & 572 & 30.0 & 1668 & 33.2 & 394 & 26.0 & 2634 \\
\hline$\geq 45$ & 32.2 & 1093 & 16.2 & 903 & 17.6 & 209 & 21.7 & 2205 \\
\hline \multicolumn{9}{|c|}{ Registration year ${ }^{* * *}$} \\
\hline 2008 & 40.3 & 1367 & 45.2 & 2516 & 11.9 & 141 & 39.7 & 4024 \\
\hline 2009 & 31.1 & 1055 & 29.6 & 1645 & 38.0 & 451 & 31.0 & 3151 \\
\hline 2010 & 28.7 & 973 & 25.2 & 1400 & 50.1 & 595 & 29.3 & 2968 \\
\hline \multicolumn{9}{|l|}{ p $<0.05$} \\
\hline $\mathrm{p}<0.01$ & & & & & & & & \\
\hline
\end{tabular}

Trop Med Int Health. Author manuscript; available in PMC 2013 September 10. 
Table 2

Differences in proportions between pediatric TB-only, TB/HIV, and TB/ART patients under age 15

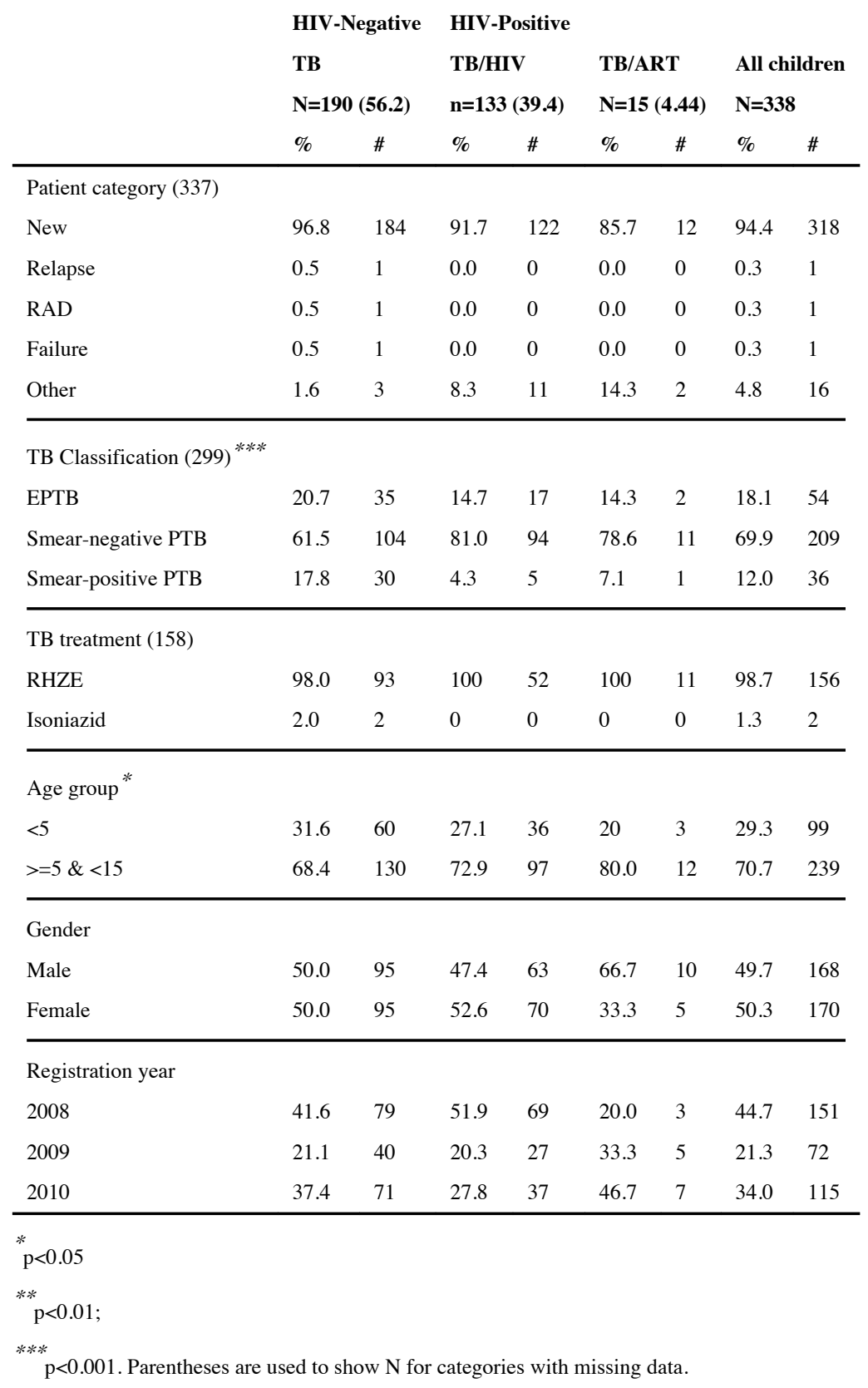

Trop Med Int Health. Author manuscript; available in PMC 2013 September 10. 


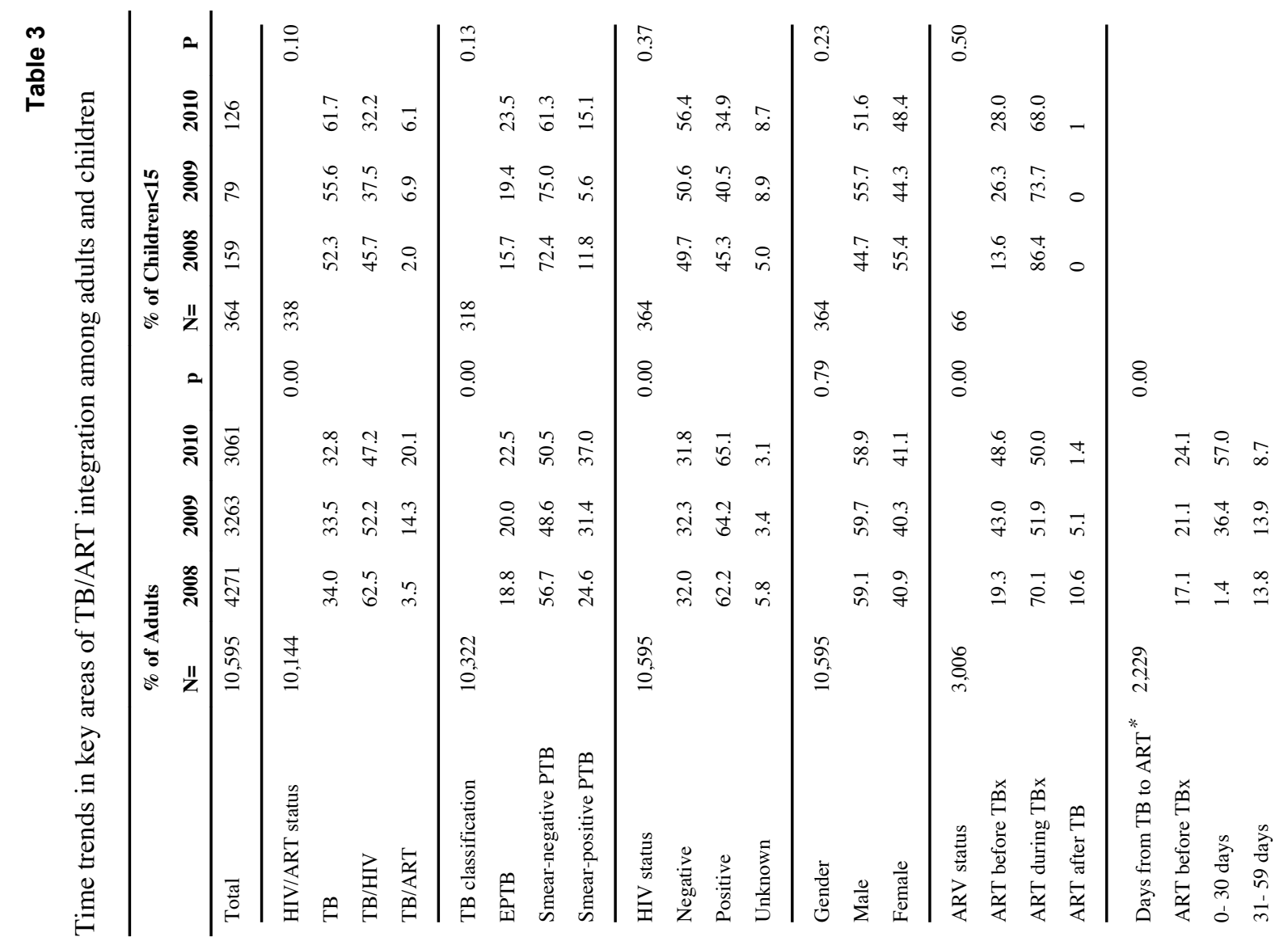




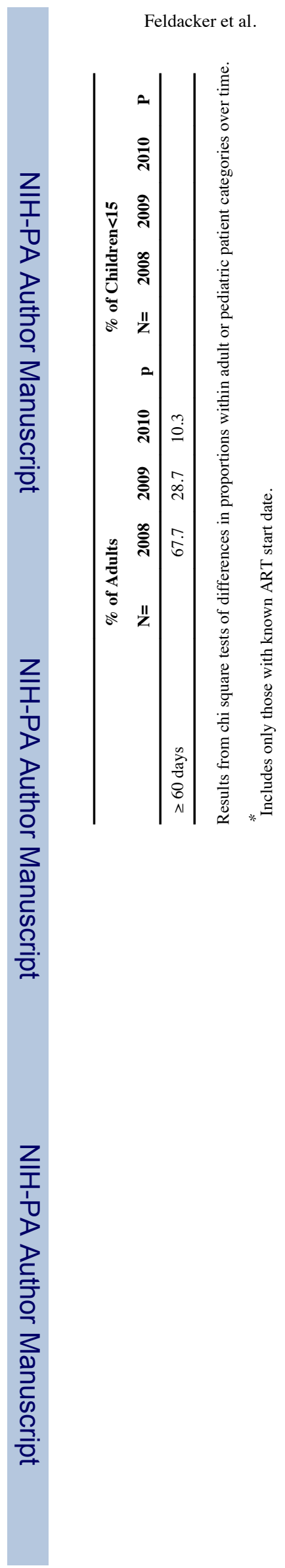

Trop Med Int Health. Author manuscript; available in PMC 2013 September 10. 